\title{
L'organisation virtuelle: un avenir qui se dessine
}

\author{
L'organisation virtuelle \\ apparaît comme une \\ réponse aux besoins de \\ flexibilité et de prompte \\ réactivité qu'éprouvent les \\ entreprises pour rester \\ compétitives. Cet article \\ tente d'élucider, grâce à \\ une revue synthèse et une \\ analyse de la littérature, les \\ éléments fondamentaux \\ reliés à ce nouveau design \\ organisationnel. L'objectif \\ étant de mieux comprendre \\ cette nouvelle forme \\ d'organisation et de mieux \\ canaliser les recherches \\ futures dans le domaine.
}

a publication, en 1992, du livre The virtual cor-
poration, par deux auteurs considérés alors
comme des futuristes, W. Davidow et M. Malone, a marqué le début d'une ère nouvelle pour l'étude des organisations. Depuis, une assertion, soutenue par un nombre sans cesse grandissant de gestionnaires praticiens, s'est progressivement répandue dans la littérature: si le $\mathrm{XXI}^{\mathrm{e}}$ siècle sera celui de l'extension des potentialités des technologies de l'information et de la communication (Ettighoffer et Van Beneden, 2000), du développement des compétences-clés (Prahalad et Hamel, 1990) et de la nécessité de partage des risques et de la saisie rapide de nouvelles opportunités offertes par de nouveaux marchés (Hutt et al., 2000), alors l'organisation virtuelle peut être attendue comme l'une des formes organisationnelles à succès des années à venir.

Le phénomène est certes nouveau, la littérature est bien à la phase de germination et les expériences pratiques sont pour le moment circonscrites aux pays industrialisés où les conditions technologiques et économiques sont propices au développement de cette forme d'organisation. Toutefois, et en dépit de l'état embryonnaire des connaissances sur le modèle de l'organisation virtuelle, plusieurs chercheurs et analystes sont convaincus que, grâce à sa flexibilité et à son agilité, ce modèle sera 
un des plus adaptés aux exigences des organisations du nouveau millénaire.

«L'entreprise virtuelle est une solution très prometteuse pour le développement général des organisations et de l'économie » affirment Ettighoffer et Van Benden (2000, p. 31). Weisenfeld et al. (2001) précisent davantage l'apport du modèle de l'organisation virtuelle et argumentent dans ce sens qu'il permettra aux entreprises de faire face à l'intensification de la concurrence en matière de coûts, de qualité et de pressions temporelles et de supporter des conditions internes et un environnement incertains. Par ailleurs, Gartner Group, une entreprise spécialisée dans les recherches et les études prévisionnelles, arrive dans l'une de ses études à la conclusion que d'ici 2005 , et avec une probabilité de $70 \%$, les organisations qui s'intégreront virtuellement domineront $70 \%$ de toutes les industries de produits et services (Joukhadar, 2000). Aussi, selon une étude conduite par Andersen Consulting et The Economist Intelligence Unit, deux chefs d'entreprises sur cinq dans le monde considèrent qu'ils devront s'organiser en entreprise virtuelle d'ici $2010^{1}$.

Toutefois, force est de constater qu'après une décennie de recherches, l'ambiguité règne toujours sur la définition même de ce nouveau mode d'organisation et sur ses principes de fonctionnement. Parce que le concept est encore nouveau et parce qu'il est difficile à délimiter, les discours sur l'organisation virtuelle se trouvent marqués par la fragmentation et le désordre. Cet état d'effritement qui caractérise la littérature jusque-là développée crée davantage de confusions que d'enseignements quant à la compréhension de ce modèle organisationnel naissant.

Devant la nébulosité du concept, nous nous proposons dans cet article de démêler ce que l'on peut qualifier aujourd'hui comme le phénomène de l'organisation virtuelle. Notre objectif étant de mieux comprendre ce modèle organisationnel émergeant et de développer un cadre de référence permettant de mieux canaliser les recherches futures. Pour ce faire, nous essayerons d'abord de caractériser, à partir d'une revue synthèse et d'une analyse de la littérature, des différentes formes organisationnelles que les auteurs qualifient d'organisations virtuelles. Nous procèderons ensuite à une comparaison des conceptions ainsi dégagées afin de mieux comprendre les spécificités de chacune d'elles. Ceci nous permettra de discuter enfin des défis que ce design organisationnel introduira sur la scène managériale.

\section{I. - L'ORGANISATION VIRTUELLE: UN CONCEPT CAMÉLÉON}

Malgré l'importance du phénomène et l'intérêt de plus en plus marqué de la part des chercheurs pour l'étude de cette nouvelle forme d'organisation (Wassenaar, 1999), la recension des travaux consacrés aux organisations virtuelles permet de constater l'absence d'une définition et d'une compréhension commune du concept. Dans les ouvrages comme dans les articles scientifiques, dans les journaux comme sur l'internet, on parle de plus en plus d'écoles et d'universités virtuelles, de bibliothèques virtuelles, de banques virtuelles et de plusieurs autres institutions qui s'apprêtent à

1. Étude Vision 2002 menée en 1997 dans 16 pays d'Europe, 13 pays d'Amérique et 5 pays d'Asie Pacifique (citée dans Ettighoffer et Van Beneden, 2000). 
prendre le virage du virtuel. Plusieurs entreprises, à l'instar de Nike, Amazon.com, Airbus, Dell, etc., sont aussi présentées dans les recherches comme des exemples typiques de firmes (ré)organisées virtuellement. Pourtant, l'examen minutieux de ces entreprises montre que des différences fondamentales les séparent.

Notre revue des principales recherches publiées sur l'organisation virtuelle permet de constater que cette expression a été utilisée pour désigner au moins cinq formes différentes d'organisation que nous examinerons dans ce qui suit.

\section{L'organisation virtuelle comme une techno-entreprise}

Le modèle de l'organisation virtuelle est apparu initialement suite aux développements qu'ont connus les technologies d'information et de communication durant les dernières années. C'est en ce sens que cer- tains auteurs (e.g. Ince, 2002; Skyrme, 1998; Rayport et Sviokla, 1995), définissent l'organisation virtuelle comme une entreprise qui utilise intensivement les nouvelles technologies d'information et de communication (NTIC) ou la technologie de façon générale pour apporter efficacité et efficience à sa chaîne de valeur. Porter (1985) résume la chaîne de valeur d'une entreprise en deux groupes d'activités: les activités principales (logistique interne, production, logistique externe, ventes et services) et les activités de support (services administratifs et de gestion, gestion des ressources humaines, développement technologique et approvisionnement). Une techno-entreprise consiste donc en une organisation qui est capable d'intégrer, d'institutionnaliser et de rentabiliser l'utilisation des NTIC au niveau de ses différentes activités principales et de support. La figure 1 donne quelques exemples de NTIC

\begin{tabular}{|c|c|c|}
\hline & Figure 1 \\
L'ORGANISATION VIRTUELLE COMME UNE TECHNO-ENTREPRISE
\end{tabular}


pouvant être utilisées dans les organisations pour y arriver.

Wal-Mart, le géant américain du commerce de détail, représente un exemple de technoentreprise. D'un simple et unique magasin en 1962 à un réseau de plus de 3000 points de vente aux États-Unis et 1000 au-delà des frontières américaines, l'évolution de l'entreprise est remarquable. Wal-Mart doit cette évolution essentiellement à une technique logistique que les dirigeants de l'entreprise ont su exploiter avec succès : l'arrimage croisé ${ }^{2}$ (Stalk et al., 1992). Grâce à ce système logistique innovateur, la marchandise reçue aux entrepôts de l'entreprise est étiquetée et acheminée aux camions pour être livrée aux magasins sans aucun séjour en stock. Dans la moitié des cas, l'opération ne dure qu'une dizaine de minutes (Bérard, 2002). Ce système a permis à Wal-Mart de réduire considérablement ses coûts de stockage et de maintenance, de profiter des économies générées par l'achat en grandes quantités et de poursuivre ainsi avec succès sa politique bien connue des «bas prix de tous les jours ».

Pour mettre en place un tel système, les dirigeants de Wal-Mart ont dû repenser le système de communication de l'entreprise. C'est ainsi, qu'un système privé de communication par satellite a été mis en place permettant de relier toutes les parties de la chaîne logistique de Wal-Mart (fournisseurs, entrepôts, points de vente, etc.) et d'assurer une rapidité extrême (souvent en quelques heures) à la saisie, la consolidation et l'exécution des ordres d'approvisionnement reçus dans les centres de distribution. De même, une grande partie des communications entre les gestionnaires tra- vaillant dans des locaux éloignés est assurée par les NTIC; les courriels et les vidéoconférences notamment. Ceci leur permet d'échanger en temps réel des informations sur le comportement du consommateur, l'efficacité des promotions ainsi que d'autres points relatifs à la gestion de l'entreprise (Stalk et al., 1992). Wal-Mart a également attaqué le lucratif marché du commerce électronique en ouvrant sa propre fenêtre sur le web (www.walmart.com).

\section{L'organisation virtuelle comme une télé-entreprise}

Pour certains auteurs (e.g. Fulton 2002; Shen, 2000, Wiesenfeld et al., 1998; Ahuja et Carley, 1998; Magretta, 1998), une organisation virtuelle est avant tout une entreprise qui utilise intensivement le télétravail. C'est d'ailleurs par analogie à ce concept que nous appelons cette conception de l'organisation virtuelle une télé-entreprise (figure 2).

Grâce aux NTIC (téléphones mobiles, télécopieurs, courriel, vidéo-conférences, etc.), les employés ne sont plus obligés de travailler dans les locaux physiques de l'entreprise. Ils peuvent travailler à partir de leurs domiciles, des locaux des clients, de ceux des fournisseurs ou encore d'un télécentre (Wiesenfeld et al., 1998). Leur local de travail devient ainsi virtuel du fait qu'il correspond, en définitive, à tout endroit où ils peuvent connecter leur modem (Skyrme, 1998). Cette forme d'organisation permet de repousser les frontières physiques de l'entreprise. Celle-ci apparaît ainsi comme une organisation qui transcende les barrières traditionnelles de la chaîne de création de valeur entre les fournisseurs, les

2. Traduction libre de l'expression anglo-saxonne « cross-docking ». 


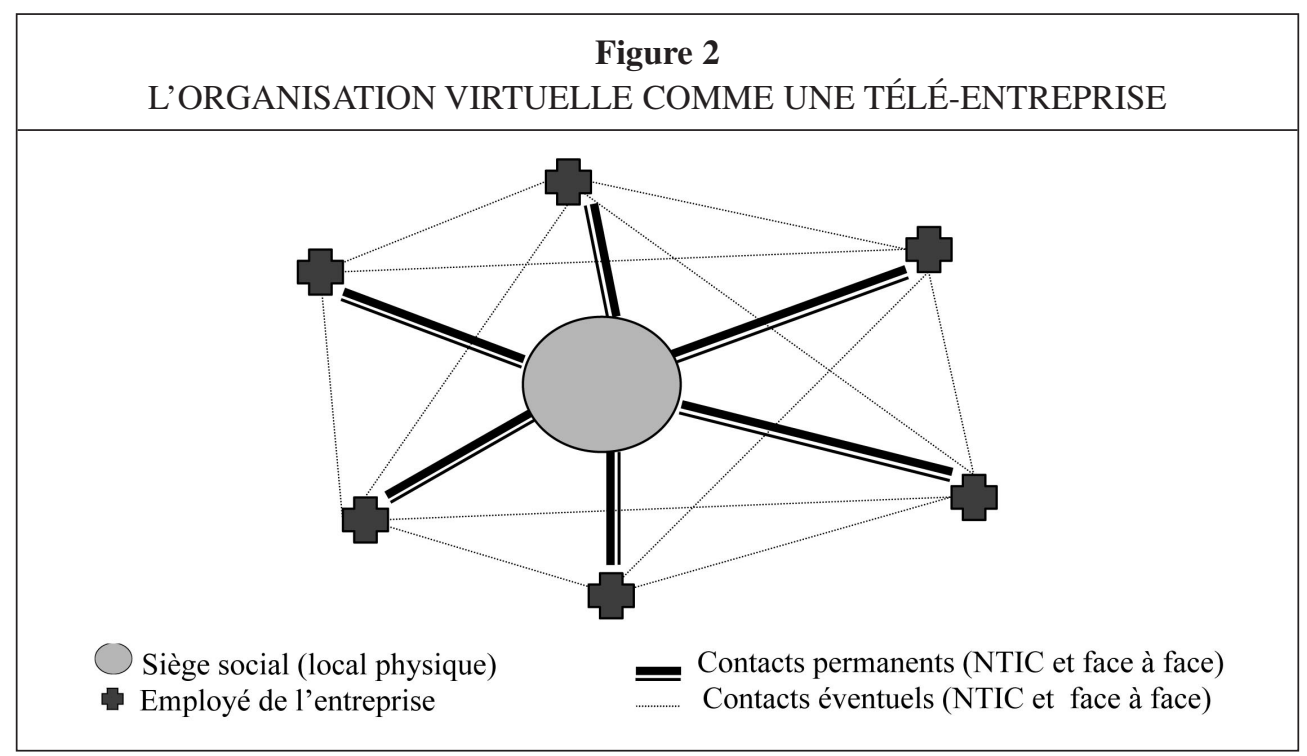

producteurs et les utilisateurs finaux du produit/service (Magretta, 1998) et où le travail est largement décentralisé (Lucas et Baroudi, 1994).

Toutefois, malgré leur dispersion, les employés de l'organisation restent liés par des intérêts durables. En effet, bien que l'organisation du travail soit nouvelle, les employés font toujours partie d'une entreprise qui possède des stratégies à court, à moyen et à long terme. La pérennité de la relation fait que ces employés auront quelques fois l'occasion de se rencontrer dans des ateliers ou des groupes de travail (Ahuja et Carley, 1998) ou encore lors des activités sociales de l'entreprise.

VeriFone, un fournisseur mondial de solutions pour sécuriser les paiements électroniques chez les institutions financières et les marchants, est l'une des organisations qui répond parfaitement aux critères de la virtualité selon cette deuxième conception. En 1997, alors filiale de Hewlett-Packard ${ }^{3}$, VeriFone comptait à son actif plus de 3300 personnes à travers le monde avec seulement moins de $7 \%$ des employés travaillant au siège social de l'entreprise situé en Californie (Boudreau et al., 1998).

Au début des années 1990, le manufacturier d'ordinateurs Compaq a également profité d'une campagne de restriction budgétaire pour passer vers ce mode d'organisation. Compaq a procédé à la fermeture des bureaux des vendeurs qui travaillent désormais chez eux tout en étant reliés au réseau de l'entreprise. Un ordinateur et un modem étaient tout ce dont les vendeurs avaient besoin. Ce faisant, Compaq a réussi à réduire son personnel administratif du tiers, à couper dans les frais de bureau et à doubler ses revenus en deux ans, avec un chiffre d'affaires par

3. Vérifone a été achetée le 20 juillet 2001 par le Gores Technology Group. 
vendeur six fois plus élevé qu'auparavant (Penzias, 1995).

\section{L'organisation virtuelle comme une entreprise externalisée}

À la différence des conceptions précédentes, pour certains auteurs (e.g. Leach 2002; Keenan et Ante, 2002; Porter, 2000; Kraut et al., 1998; Palmer et Speier, 1997), le degré de virtualité d'une organisation dépend essentiellement de la masse des fonctions externalisées (i.e. confiées à des entreprises spécialisées). La virtualité est ainsi perçue comme un continuum où les entreprises deviennent de plus en plus virtuelles à mesure qu'elles sous-traitent des activités supplémentaires de leur chaîne de création de valeur (figure 3). L'adoption de ce mode d'organisation vise l'optimisation de la chaîne de valeur de l'entreprise. À l'extrême de cette conception, une organisation est considérée comme totalement virtuelle si chacune des étapes de produc- tion de ses biens ou services est accomplie à l'extérieur de l'entreprise. Celle-ci jouera alors le rôle de coordinateur de ces différentes étapes (Kraut et al., 1998). Les fonctions externalisées sont souvent des fonctions «périphériques ». Les fonctions-clés, sur lesquelles repose l'avantage concurrentiel de l'entreprise, sont souvent gardées à l'interne.

Le manufacturier d'articles de sport américain Nike est un bon exemple d'entreprise externalisée. Nike s'est engagée depuis quelques années dans un mouvement d'externalisation sans précédent. Les fonctions de production, de distribution, de marketing et de vente au détail sont actuellement toutes sous-traitées. À l'interne, Nike se contente d'assurer la seule gestion de sa marque (Finance Week, 1998). Sun Microsystems est un autre exemple d'entreprise externalisée. Le siège social de l'entreprise n'assure plus que la fonction de design des ordinateurs qu'elle offre. Toutes les autres

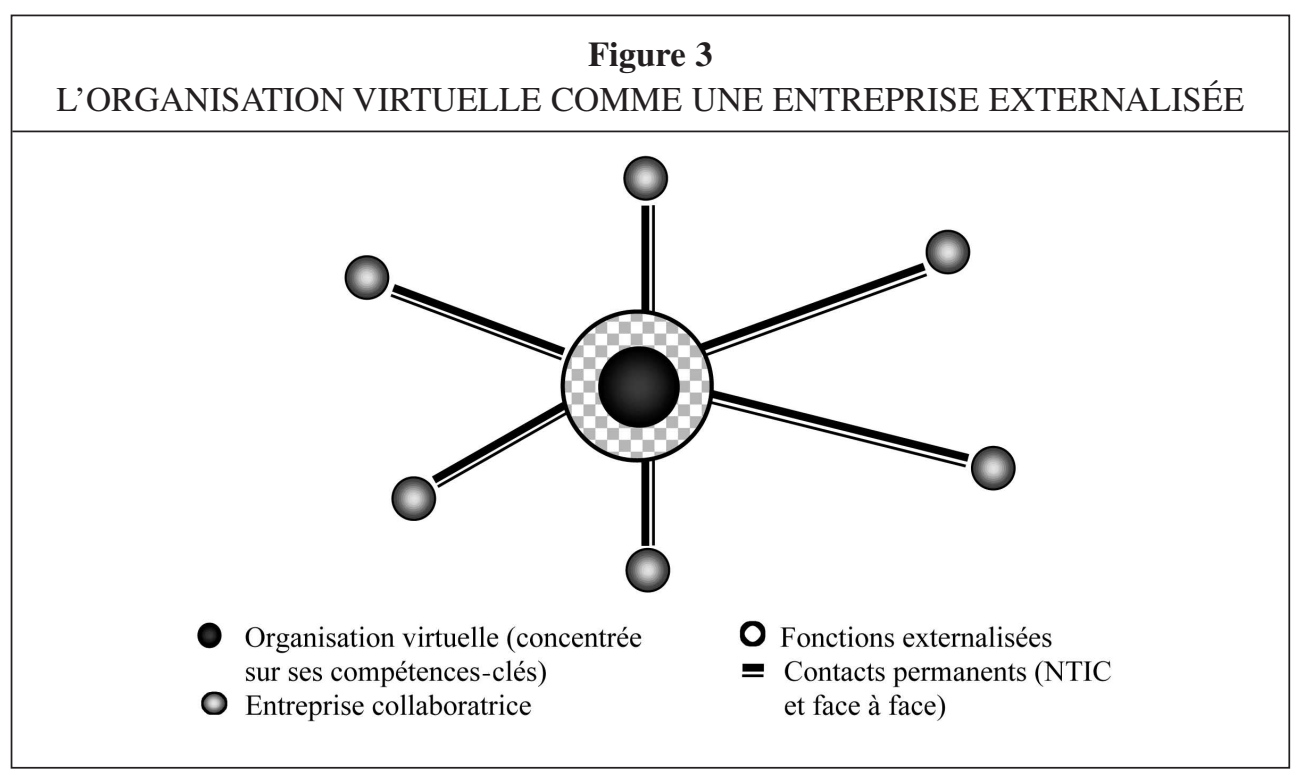


fonctions de collecte des commandes, de production, de distribution, de marketing et de service à la clientèle sont réalisées par des arrangements contractuels avec des partenaires à travers le monde (Boudreau et al., 1998).

\section{L'organisation virtuelle comme une cyberentreprise}

Lipnack et Stamps (1997) définissent une cyberentreprise comme une interconnexion de personnes ou de groupes indépendants. La mission de l'entreprise se résume à la collecte des commandes et à la coordination de la livraison du produit/service au client. Tout ceci se faisant dans le cyberespace (i.e. l'univers créé par l'internet); d'où d'ailleurs son appellation de cyberentreprise (figure 4). C'est, en définitive, une simple carapace qui assure la liaison entre les producteurs et le client final. L'essor considérable d'internet a eu un véritable effet de catalyseur pour le développement des cyberentreprises et ce, par la création d'un univers virtuel auquel des millions d'utilisateurs à travers le monde peuvent régulièrement, simultanément et à tout moment être connectés.

Ainsi définie, une cyberentreprise correspond à une situation quasi totalement immatérielle. À la limite, une cyberentreprise n'a besoin ni d'immobilisations centralisées, ni d'usines, et c'est ce qui la différencie des organisations classiques (Hartman et Guss, 1996). Avec le modèle des cyberentreprises, les barrières à l'entrée se trouvent abaissées dans plusieurs industries (Loebbecke et Troelsen, 2000). Ceci vient refaçonner les règles de la concurrence à l'échelle mondiale. Là où le marketing traditionnel favorise les grandes entreprises, le marketing via l'internet profite à toutes les firmes quelle que soit leur taille. Les cyberentreprises n'ont pas besoin d'être grandes pour réussir. Grâce à ses avantages au niveau des coûts et de la flexibilité, le modèle des cyberentreprises connaît un succès sans cesse grandissant auprès des dirigeants et des jeunes

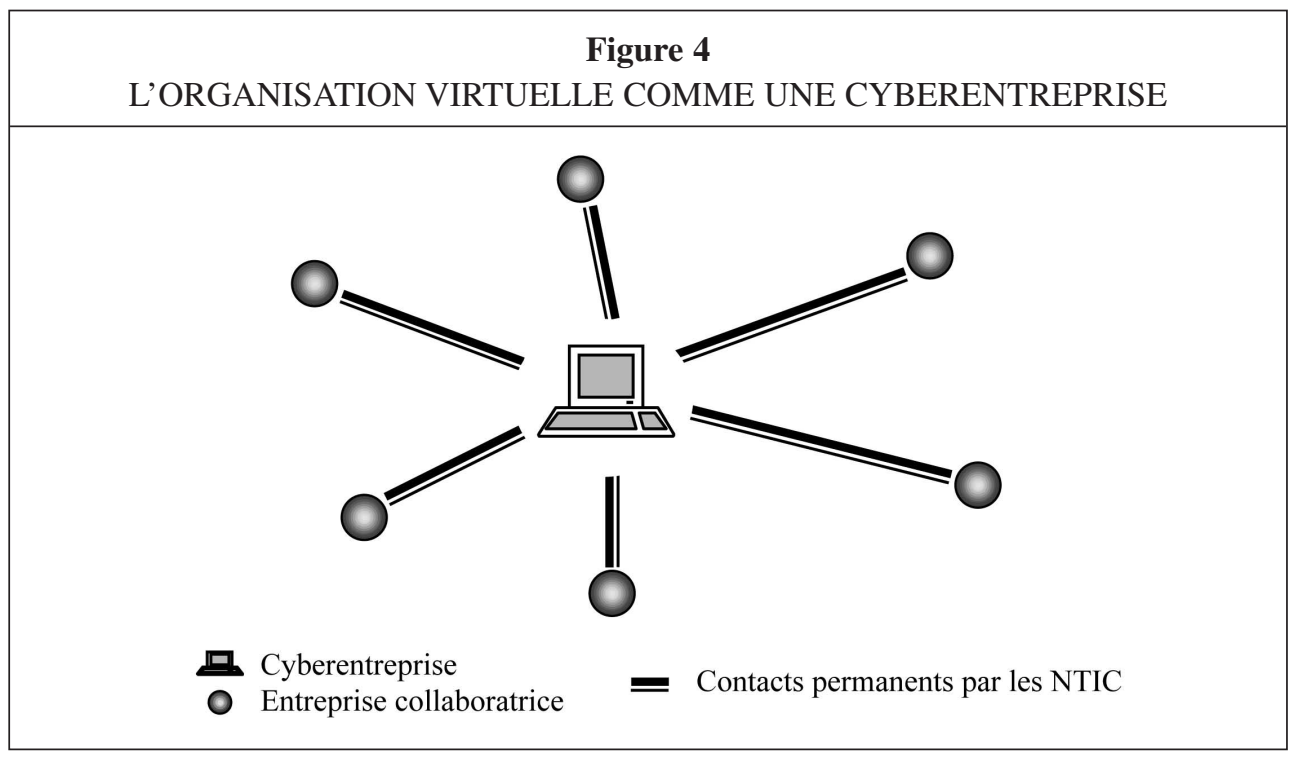


entrepreneurs. Nous citerons, pour fin d'illustration, l'entreprise eBay comme un exemple typique de cyberentreprise. eBay a ouvert ses portes « virtuelles » en septembre 1995 avec pour mission d'aider les gens à commercer, via l'internet, à peu près tout ce qui peut être transigé. Le concept était à la fois innovateur et attrayant ce qui lui a valu les intérêts de plusieurs millions d'individus et d'un nombre élevé d'entreprises à travers le monde.

En 2002, la communauté de eBay se chiffrait à 49,7 millions de membres enregistrés et son volume de transaction s'élevait à plusieurs milliards de dollars, ce qui en fait l'un des plus importants marchés virtuels au monde. Pourtant, eBay ne possède ni un grand siège social au sens classique du terme, ni immobilisations, ni usines de production. Si vous désirez faire des affaires avec eBay, personne ne vous référera à son local physique situé en Californie, comme on ne vous donnera pratiquement jamais un numéro de téléphone ou de fax. Vous serez référés à une seule adresse, à un seul cyberendroit: http://www.ebay.com

\section{L'organisation virtuelle comme un réseau temporaire}

La dernière conception de l'organisation virtuelle identifiée dans la littérature est ce que nous appellerons le réseau temporaire (figure 5). Pour les adeptes de cette conception, une organisation virtuelle consiste en un groupe d'entreprises formé autour d'un projet commun et qui, une fois le projet terminé, sera dissout permettant à chaque membre de participer à d'autres projets (Morris et al., 2002; Franke et Hickmann, 1999; Bultje et Van Wijk, 1998; Amherdt et Su, 1997; Wigand et al., 1997; Byrne et al., 1993). Le réseau temporaire est un peu comme une molécule qui se fait et se défait à mesure que des atomes différents s'y greffent (Davidow et Malone, 1992).

La marque fondamentale de cette conception de l'organisation virtuelle est la temporalité perçue dans une perspective de réseau.

Figure 5

L'ORGANISATION VIRTUELLE COMME UN RÉSEAU TEMPORAIRE

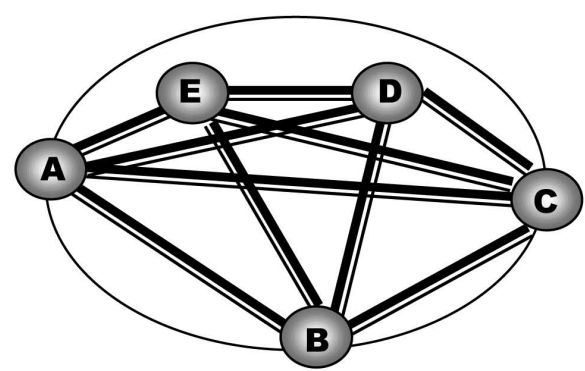

Organisation virtuelle (réseau)

Entreprise membre (composante Contacts fréquents par les NTIC du réseau) 
L'expression « organisation virtuelle » fait ainsi référence au groupe temporaire formé par deux ou plusieurs entreprises qui seront quant à elles considérées comme des membres ou des composantes du groupe (Jones et Bowie, 1998; Dembski, 1998). Dans certains cas, - et c'est souvent le cas des PME -, toute l'entreprise sera engagée dans l'association (cas des entreprises $E$ et D dans la figure 5). Dans d'autres cas, l'entreprise peut s'impliquer en partie en engageant uniquement une de ses compétences distinctives (cas des entreprises A, B et C dans la figure 5). Notons également qu'une entreprise peut être engagée dans plusieurs réseaux temporaires à la fois.

Les membres d'un réseau temporaire fonctionnent sur une même plate-forme informationnelle. Ils sont interreliés par les NTIC et les personnes travaillant ensemble dans un même projet ont rarement l'occasion de se rencontrer face-àface (Fritz et Manheim, 1998; Jones et Bowie, 1998; Knoll et Jarvenpaa, 1995). Le processus de production du bien ou service, qui transcende les barrières de chacune des entreprises membres du réseau, ne peut être contrôlé par l'une d'entre elles (Kraut et al., 1998). Par conséquent, la structure de l'organisation virtuelle est principalement formée par des nœuds (entreprises membres) et des interconnexions (contacts électroniques). Cette forme de coopération est très flexible et permet une minimisation considérable des coûts de production ainsi qu'une rationalisation de l'utilisation des ressources disponibles pour chacune des entreprises participantes (Snow et al., 1996).
Cette conception de l'organisation virtuelle met l'accent sur la rapidité de formation, de réorganisation et de dissolution du groupe (Kristof et al., 1995; Christie et Levery, 1998). Les composantes du réseau virtuel sont souvent réunies autour d'un objectif ou d'un intérêt commun de court terme. De plus, un réseau virtuel peut être constitué par des membres qui n'ont jamais travaillé ensemble dans le passé et qui ne travailleront pas nécessairement ensemble dans l'avenir (Jarvenpaa et Ives, 1994; Lipnack et Stamps, 1997). Ces membres peuvent même devenir concurrents dans un projet ultérieur (Christie et Levery, 1998).

Le modèle du réseau temporaire connaît un essor particulier en Europe notamment. Les projets réalisés conformément à ce mode d'organisation sont de plus en plus nombreux. Blis, GlobeMen, Procure et ICCI sont les noms de certains projets en cours alors que Combine, Rinet, Connet et I-SEEK sont les noms de certains projets récemment terminés ${ }^{4}$.

\section{II. - COMPARAISON DES CINQ CONCEPTIONS DE L'ORGANISATION VIRTUELLE}

Pour mieux comprendre chacune des cinq conceptions de l'organisation virtuelle, nous procéderons à leur comparaison selon cinq caractéristiques fondamentales (tableau 1). Notons que ces caractéristiques ne procurent pas un cadre exhaustif pour comparer ces différentes visions de l'organisation virtuelle. Néanmoins, leur distinction est assez pertinente pour apprécier les fondements et les présuppositions théo-

4. Pour les détails sur ces projets consulter l'adresse URL suivante: http://cic.vtt.fi/projects/ 
riques soutenant les différentes formes de ce nouveau modèle organisationnel.

Tout d'abord, la composition de l'entreprise nous permet de séparer le réseau temporaire des quatre autres conceptions (i.e. la techno-entreprise, la télé-entreprise, l'entreprise externalisée et la cyberentreprise) et ce, à deux niveaux :

- les quatre premières conceptions, l'entreprise virtuelle est formée par des individus (ses employés) alors que le réseau temporaire est constitué par des entreprises;

- dans un réseau temporaire, le caractère éphémère de la relation et la nature même de l'association font qu'il n'est pas nécessaire que les membres aient des expériences partagées avant ou après leur alliance virtuelle. Du côté des quatre autres conceptions par contre, le fait que les membres de l'organisation virtuelle soient ses propres employés laisse les chances de l'existence d'un passé et d'un avenir commun entre ces membres quasi identiques au cas des organisations classiques.

Les cinq conceptions de l'organisation virtuelle sont ensuite différentes en ce qui concerne les interactions interfirmes qu'elles impliquent. Si les deux premières conceptions définissent la virtualité par rapport à une entreprise confinée, les trois autres ne l'envisagent qu'en considération d'un réseau plus ou moins complexe de

Tableau 1

COMPARAISON DES CINQ CONCEPTIONS DE L'ORGANISATION

\begin{tabular}{|c|c|c|c|c|c|}
\hline & $\begin{array}{l}\text { La techno- } \\
\text { entreprise }\end{array}$ & $\begin{array}{c}\text { La } \\
\text { télé-entreprise }\end{array}$ & $\begin{array}{l}\text { L'entreprise } \\
\text { externalisée }\end{array}$ & $\begin{array}{c}\text { La } \\
\text { cyberentreprise }\end{array}$ & $\begin{array}{l}\text { Le réseau } \\
\text { temporaire }\end{array}$ \\
\hline \multicolumn{6}{|l|}{$\begin{array}{l}\text { Composition } \\
\text { de l'entreprise }\end{array}$} \\
\hline Membres de l'entreprise & Employés & Employés & Employés & Employés & Employés \\
\hline $\begin{array}{l}\text { Existence d'un passé } \\
\text { et d'un avenir commun } \\
\text { entre les membres }\end{array}$ & Oui & Oui & Oui & Oui & Pas nécessairement \\
\hline \multicolumn{6}{|l|}{$\begin{array}{l}\text { Interactions } \\
\text { interfirmes }\end{array}$} \\
\hline Existence d'un réseau & Non & Non & Oui & Oui & Oui \\
\hline $\begin{array}{l}\text { Existence d'une } \\
\text { entreprise noyau }\end{array}$ & N/A & N/A & Oui & Oui & Oui \\
\hline Rapports de force & N/A & N/A & Asymétriques & Asymétriques & Symétriques \\
\hline \multicolumn{6}{|l|}{$\begin{array}{l}\text { Contours } \\
\text { de l'entreprise }\end{array}$} \\
\hline $\begin{array}{l}\text { Existence d'un lieu } \\
\text { physique }\end{array}$ & Oui & Oui & Oui & $\begin{array}{c}\text { Pas nécessairement } \\
\text { (Existence } \\
\text { cybernétique) }\end{array}$ & Non \\
\hline Frontières & Traditionnelles & $\begin{array}{c}\text { Géographiquement } \\
\text { élargies }\end{array}$ & $\begin{array}{c}\text { Géographiquement } \\
\text { rétrécies }\end{array}$ & Virtuelles & $\begin{array}{c}\text { Géographiquement } \\
\text { confuses }\end{array}$ \\
\hline $\begin{array}{l}\text { Durée de vie } \\
\text { de l'entreprise }\end{array}$ & $\begin{array}{c}\text { Relativement } \\
\text { longue }\end{array}$ & $\begin{array}{l}\text { Relativement } \\
\text { longue }\end{array}$ & $\begin{array}{l}\text { Relativement } \\
\text { longue }\end{array}$ & À suivre ! & $\begin{array}{l}\text { Relativement } \\
\text { courte }\end{array}$ \\
\hline $\begin{array}{l}\text { Support } \\
\text { communicationnel }\end{array}$ & NTIC et FF & NTIC et FF & NTIC et FF & $\begin{array}{l}\text { Exclusivement } \\
\text { NTIC }\end{array}$ & $\begin{array}{l}\text { Principalement } \\
\text { NTIC }\end{array}$ \\
\hline
\end{tabular}

N/A = non applicable. 
relations interfirmes. De plus, dans le cas de l'entreprise externalisée et de la cyberentreprise, le réseau est toujours mené par un noyau qu'est l'entreprise virtuelle ellemême. Ceci se traduit par des rapports de forces asymétriques entre les entreprises membres du réseau et ce, en faveur de l'entreprise noyau. À l'opposé, les membres d'un réseau temporaire participent au projet sur le même pied d'égalité. Par conséquent, aucun noyau n'est distingué et les rapports de force entre les partenaires sont, en principe, symétriques (Jägers et al., 1998).

Par ailleurs, s'il est vrai que le modèle de l'organisation virtuelle vient changer fondamentalement les frontières traditionnelles de la firme (à l'exception de la technoentreprise où les frontières traditionnelles demeurent), ce changement reste assez différent d'une conception à l'autre. Pour la télé-entreprise, l'utilisation du télétravail engendre un élargissement des frontières de l'organisation par rapport à celles de l'organisation classique. À l'inverse, les frontières de l'entreprise externalisée sont rétrécies à cause du mouvement potentiel d'externalisation auquel procède la firme. Dans le cas de la cyberentreprise, les frontières de la firme deviennent carrément virtuelles du fait que son existence est essentiellement cybernétique. Quant au réseau temporaire, les frontières de l'organisation virtuelle sont plutôt confuses. En effet, la collaboration étroite entre les différentes entreprises membres du réseau virtuel rend difficile la détermination des frontières du réseau dans son ensemble.

Les cinq conceptions de l'organisation virtuelle se distinguent ensuite par la durée de vie de l'entreprise. Le réseau temporaire, nous l'avons vu, est constitué sur une base opportuniste. Sa durée de vie est relativement courte puisqu'elle est limitée à la durée de réalisation du projet pour lequel il est formé. Pour les trois premières conceptions, la virtualité vient surtout revoir et redéfinir la manière de faire les choses à l'intérieur de l'entreprise mais la durée de vie de l'organisation reste similaire à celle des organisations classiques soit principalement le long terme ou, du moins, une durée de vie relativement plus longue que celle du réseau temporaire. Quant à la cyberentreprise, étant donnée la nouveauté du modèle, nous ne pouvons nous prononcer avec certitude quant à sa durée de vie moyenne. C'est donc un modèle organisationnel à suivre sur ce point.

Le support communicationnel représente enfin le dernier axe que nous distinguons pour discriminer les cinq conceptions de l'organisation virtuelle. Par support communicationnel, nous entendons les principaux moyens de communication utilisés par les membres de l'entreprise. Le support communicationnel permet de distinguer les trois premières conceptions des deux autres. En effet, le fait que dans les trois premières conceptions l'entreprise soit formée par ses propres employés et que la durée de vie de l'entreprise soit relativement longue fait que ces membres auront occasionnellement l'occasion de se rencontrer face-à-face. Le support communicationnel est donc formé par un mixe de NTIC et de contacts interpersonnels. Toutefois, la rapidité de formation et de dissolution du réseau temporaire fait que les contacts face-à-face ont très peu de chance d'avoir lieu entre les membres du groupe. Les communications entre ces membres se font essentiellement au moyen des NTIC. De même, dans le cas de la cyberentreprise, l'utilisation intensive des principes de la cybernétique fait que les contacts entre les 
différents membres de l'entreprise ainsi qu'avec ses partenaires et clients se font exclusivement par les NTIC.

Nous tenons à préciser à ce niveau de notre argumentation que la distinction que nous faisons entre les cinq conceptions de l'organisation virtuelle vise essentiellement à clarifier les différentes facettes du concept. Toutefois, force est de constater que si cette étape représente un pas important vers la compréhension de ce nouveau phénomène, il n'en demeure pas moins que la distinction à laquelle nous avons procédé reste essentiellement théorique. Dans la pratique les barrières entre ces conceptions sont assez fluides et perméables. L'entreprise Intel, par exemple, structure ses activités par projets. Les membres du groupe chargé d'un projet donné proviennent des différentes filiales de l'entreprise situées aux quatre coins du monde. Ils sont rapidement regroupés pour réaliser le projet et se séparent ensuite pour s'intégrer dans d'autres groupes formés pour d'autres projets (Boudreau et al., 1998). En considérant les différents membres participant aux projets comme des employés d'Intel, nous définirons celleci comme une télé-entreprise. En effet, bien que les groupes disparaissent après la réalisation d'un projet donné, les employés, eux, restent toujours ceux d'Intel. D'autre part, si nous considérons les différentes filiales d'Intel comme des entreprises indépendantes, tout projet réalisé par Intel serait plutôt un réseau temporaire.

\section{III. - LES DÉFIS MANAGÉRIAUX SOULEVÉS PAR LE MODÈLE DE L'ORGANISATION VIRTUELLE}

En guise de conclusion, il serait opportun, pour arguer de ce portrait des cinq concep- tions de l'organisation virtuelle, de discuter des principaux défis que le modèle de l'organisation virtuelle introduit sur la scène managériale. La gestion d'une organisation virtuelle est loin d'être facile puisqu'elle doit combiner fluidité et contrôle, différences et cohésion et, dans le cas du réseau temporaire, des relations fortes dans un partenariat éphémère. S'organiser virtuellement exige ainsi une définition et une considération particulière des problèmes organisationnels essentiels auxquels les gestionnaires seront confrontés. Nous soulèverons brièvement ces challenges managériaux que nous conceptualisons en cinq «Cs » que sont: la gestion de la codestinée, la cohésion, la gestion des compétences, la gestion du changement et le contrôle des activités (figure 6).

\section{Codestinée}

La nature de la collaboration au sein d'une organisation virtuelle implique une interdépendance élevée entre ses membres. Ceuxci partageront un avenir commun dans le sens où la destinée de chacun d'entre eux dépend en grande partie de celle des autres. La gestion de la co-destinée implique la conciliation des intérêts individuels et collectifs. Si ces intérêts étaient plus ou moins identifiables dans les organisations classiques guidées par les principes tayloriens ou weberiens de gestion, elles le sont difficilement dans le cadre des organisations virtuelles. La situation est expressément complexe dans l'entreprise externalisée, la cyberentreprise et le réseau temporaire. Dans ces trois conceptions de l'organisation virtuelle trois niveaux d'intérêts sont à réconcilier: les intérêts du groupe, les intérêts de chaque entreprise membre et les intérêts des employés de ces entreprises. 


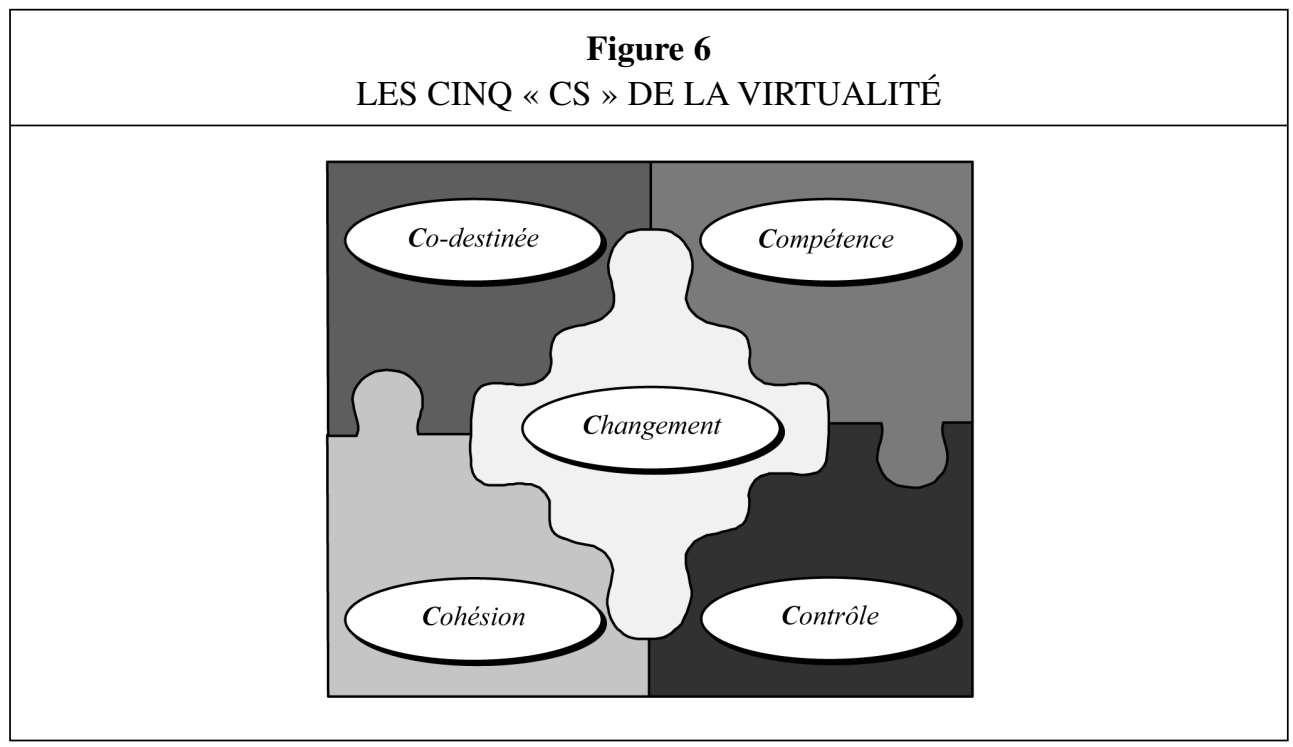

L'identification de ces trois niveaux d'intérêts et leur conciliation ne sont pas du tout évidentes ce qui fait de la gestion de la codestinée un véritable défi.

\section{Cohésion}

Le problème complexe du maintien de la cohésion de l'entreprise est très lié à la gestion de la codestinée. En effet, mis à part le cas de la techno-entreprise, la virtualité implique nécessairement une distanciation spatiotemporelle des partenaires. Cette dispersion pose un sérieux problème d'unité et d'intégration du groupe virtuel (Kugelmass, 1995). Plus précisément, le souci est celui de la fragmentation et de la dislocation de l'organisation suite à la rareté voire l'absence de rencontres interpersonnelles entre ses membres (Wiesenfeld et al., 1998; Handy, 1995). Certaines études (e.g. Jarvenpaa et Leidner, 1998; Townsend et al., 1998; Gibbs, 1998) suggèrent sur ce point que les NTIC seront le moyen d'inté- gration par excellence d'une organisation virtuelle. Toutefois, bien qu'elles soient primordiales pour assurer l'intégration de travailleurs dispersés dans le temps et dans l'espace, les NTIC ne peuvent pas à elles seules garantir le bon fonctionnement de l'organisation. Sinon, et comme l'affirment Nohria et Eccles (1992) nous aurons probablement besoin d'une sociologie complètement nouvelle des organisations.

\section{Compétences}

Le problème de la gestion des compétences se pose particulièrement dans le cas de l'entreprise externalisée, de la cyberentreprise et du réseau temporaire. Dans ces trois conceptions de l'organisation virtuelle, le principe de l'organisation est de se concentrer sur ses compétences-clés et de solliciter la collaboration d'autres entreprises pour réaliser les activités déficientes de la chaîne de valeur. Pour réussir son apparition ou sa réorganisation selon un mode virtuel, une 
organisation doit donc veiller à développer et à nourrir des compétences-clés. Mais, de l'autre côté, agir de la sorte implique nécessairement d'autres problèmes notamment au niveau de la gestion des ressources humaines. En effet, la gestion des compétences pose un sérieux problème quant à la planification de carrière pour les employés. Alors que l'organisation traditionnelle, guidée souvent par le taylorisme, offrait la sécurité d'emploi en contrepartie d'un investissement limité dans ses ressources humaines, l'organisation virtuelle se situe, à ce niveau, aux antipodes de cette dernière.

\section{Contrôle}

La question de la coordination et du contrôle devient particulièrement délicate quand elle est rattachée au contexte des organisations virtuelles (Jägers et al., 1998). En effet, établir la coordination et le contrôle devient de plus en plus difficile lorsque les membres de l'organisation sont dispersés et se voient attribuer des tâches parfois imprévisibles et dont les résultats sont difficilement mesurables (DeSanctis, 1984). Comme le soulignent avec raison Perlo et Hills (1998, p. 114): « [Les équipes virtuelles] ne sont pas seulement multisites [i.e. dispersées dans le temps et dans l'espace], elles sont multi-tout: multilingues, multiculturelles, multifonctions, multimétiers, etc. Or, quand on parle la même langue, qu'on partage le même système de valeurs et de croyances, et qu'on est tous dans le même lieu, travailler efficacement en équipe représente déjà un réel défi. Cela devient plus qu'un challenge quand les membres de l'équipe sont à 5000 kilomètres de distance, avec des décalages horaires de plusieurs heures, des problèmes de langue et des pratiques professionnelles différentes. »
La définition même du contrôle est sujette à des révisions lorsque appliquée aux organisations virtuelles. Les mécanismes traditionnels de coordination et de contrôle (supervision directe, renforcement des règles et des procédures, etc.) sont difficilement applicables et souvent inefficaces dans un contexte où les partenaires sont dispersés (Blake et Suprenant, 1990). Désormais, le système « ordonne-contrôle » ne fonctionne plus et l'autonomie de décision et la décentralisation s'imposent. Les organisations virtuelles auront donc besoin de mécanismes de contrôle informels, soft et flexibles, faisant très peu appel aux procédures et aux règles formelles.

\section{Changement}

Le changement a toujours été un problème majeur pour les gestionnaires. Il exige beaucoup de réflexion, une bonne planification, une démarche inhabituelle et un leadership à toute épreuve. Sa réalisation mobilise les énergies tout en déclenchant de fortes résistances. Or, pour une organisation virtuelle, le changement devient l'essence même de son existence. Une organisation virtuelle se doit d'être agile, flexible et apprenante. Elle doit être une molécule, une sorte de «pâte à modeler » en continuelle adaptation à son environnement. Elle doit constamment apprendre à apprendre, se remettre en question et surtout ne rien prendre pour acquis. Le défi pour les gestionnaires des organisations virtuelles est celui d'arriver à gérer et à mobiliser toutes les conditions de réussite d'un changement au quotidien.

Notre discussion permet ainsi de constater que les cinq formes organisationnelles que les auteurs qualifient d'organisations virtuelles correspondent toutes à des situations 
assez novatrices par rapport aux organisations classiques mais aussi assez différentes l'une de l'autre. Naturellement, ces conceptions soulèvent, à des degrés différents, plusieurs défis auxquels les gestionnaires d'une organisation virtuelle seront confrontés. Comment relever ces défis? C'est certainement la réponse à cette question qui déterminera le sort de ce nouveau modèle organisationnel.

\section{BibLIOGRAPHIE}

M. K. Ahuja, K. M. Carley, "Network Structure in Virtual Organizations", Journal of Computer-Mediated Communication, $\mathrm{n}^{\circ} 3(4)$, http://www.ascusc.org/jcmc/vol3/issue4/ ahuja.html, 1998.

C. H. Amherdt, Z. Su, « Vers une gestion renouvelée des ressources humaines dans les organisations virtuelles », Revue de Gestion des Ressources Humaines, ${ }^{\circ}{ }^{23}$, 1997, p. 14-26.

D. Bérard, « Super Mario », Commerce, vol. 103, n 11, 2002, p. 28-31.

V. L. P. Blake, T. T. Suprenant, "Electronic immigrants in the information age: Public policy considerations", The Information Society, n 7, 1990, p. 233-244.

M. C. Boudreau, K. D. Loch, D. Robey, D. Straud, "Going Global: Using information technology to advance the competitiveness of the virtual transnational organization", Academy of Management Executive, $\mathrm{n}^{\circ}$ 12(4), 1998, p. 120-128.

R. Bultje, J. Van Wijk, "Taxonomy of Virtual Organizations, Based on Definitions, Characteristics and Typology", Virtual-organization.net, Newsletter, ${ }^{\circ} 2(3), 1998$, p. 7-21, www.virtual-organization.net.

J. Byrne, R. Brandt, O. Port, "The virtual corporation", Business week, $\mathrm{n}^{\circ}$ 3304, 1993, p. 98-104.

M. J. Christie, R. R. Levary, "Virtual corporations: Recipe for success", Industrial Management, Norcross, ${ }^{\circ}$ 40(4), 1998, p. 7-11.

W. H. Davidow, M. S. Malone, The virtual corporation, New York, NY, Edward Burlingame Books/Harper Business, Harper Collins Publishers, 1992.

T. M. Dembski, "Future Present: The Concept of Virtual Organization Revisited, The Nature of Boundedness of Virtual Organizations", Virtual-organization.net, Newsletter, $\mathrm{n}^{\circ} 2(2)$, 1998, p. 37-58, www.virtual-organization.net

G. DeSanctis, "Attitudes toward telecommuting: Implications for work-at-home programs", Information \& Management, $\mathrm{n}^{\circ}$ 7, 1984, p. 133-139.

D. Ettighoffer, P. Van Beneden, Méta-organisations, Les modèles d'entreprise créateurs de valeur, Éditions Village Mondial, Paris, 2000.

Finance Week, «Welcome to virtual business », 10-16 septembre 1998, p. 42-44.

U. Franke, B. Hickmann, "Is The Net-Broker an Entrepreneur? What Role does the Net-Broker play in Virtual Webs and Virtual Corporations?", Organizational Virtualness and Electronic Commerce, P. Sieber, J. Griese (eds), Proceedings of the VoNet-Workshop, Simowa Verlag Bern, 1999, p. 120-139, www.virtual-organization.net 
M. B. Fritz, M. L. Manheim, "Managing Virtual Work: A Framework for Managerial Action”, P. Sieber, J. Griese (eds.), Organizational Virtualness, Proceedings of the VoNetWorkshop, Simowa Verlag Bern, 1998, p. 123-135, www.virtual-organization.net M. L. Fulton, "Working Virtually", SEARCHER: The Magazine for Database Professionals, April, 2002, p. 50-54.

M. Gibbs, "Managing by messaging", Network World, $\mathrm{n}^{\circ}$ 15(37), Framingham, 1998, p. $59-60$.

C. Handy, "Trust and the virtual organization", Harvard Business Review, n 73(3), 1995, p. $40-50$.

F. Hartman, C. Guss, "Virtual teams - constrained by technology or culture", Proceedings of IEMC 96, Managing virtual enterprises: A convergence of communications, computing and energy technologies, Vancouver, BC, Canada, IEEE, 1996, p. 645-650.

M. D. Hutt, E. R. Stafford, B. A. Walker, P. H. Reingen, "Case study Defining the Social Network of a Strategic Alliance", Sloan Management Review, n 41(2), 2000, p. 51-62.

J. Ince, "Catching Up With SCM's Vision", UPSIDE, July 2002, p. 50-54.

H. Jägers, W. Jansen, W. Steenbakkers, "Characteristics of Virtual Organizations", Organizational Virtualness, P. Sieber, J. Griese (eds.), Proceedings of the VoNet -Workshop, Simowa Verlag Bern, 1998, p. 65-76, www.virtual-organization.net

S. L. Jarvenpaa, B. Ives, "The global network organization of the future: Information management opportunities and challenges", Journal of Management Information Systems, $\mathrm{n}^{\circ} 10(4), 1994$, p. 25-57.

S. L. Jarvenpaa, D. E. Leidner, « Communication and Trust in Global Virtual Teams », Journal of Computer-Mediated Communication, $\mathrm{n}^{\circ} 3(4)$, 1998. http://www.ascusc.org/jcmc/ vol3/issue4/jarvenpaa.html

T. M. Jones, N. E. Bowie, "Moral hazards on the road to the 'virtual' corporation", Business Ethics Quarterly, Chicago, n 8(2), 1998, p. 273-292.

K. Joukhadar, "Virtual Enterprise Boosts Collaborative E-Commerce", Information Week, November 6, 2000, p. 146.

F. Keenan, S. E. Ante, "The New Teamwork", Business Week, EB12-EB16, February 18, 2002.

S. Klein, Interorganisationsysteme und Unternehmensnetzwerke. Wechselwirkungen zwischen organisatorischer und informationstechnischer Entwicklung, Wiesbaden, 1996.

K. Knoll, S. L. Jarvenpaa, "Learning virtual team collaboration", Hawaii International Conference on Systems Sciences Proceedings, vol. 4, 1995, p. 92-101.

R. Kraut, C. Steinfield, A. Chan, B. Butler, A. Hoag, "Coordination and Virtualization: The Role of Electronic Networks and Personal Relationships", Journal of Computer-Mediated Communication, $\mathrm{n}^{\circ}$ 3(4), 1998. http://www.ascusc.org/jcmc/vol3/issue4/kraut.html

A. L. Kristof, K. G. Brown, Jr. H. P. Sims, K. A. Smith, "The virtual team: A case study and inductive model”, M. M. Beyerlein, D. A. Johnson, S. T. Beyerlein (Eds.), Advances in interdisciplinary studies of work teams: Knowledge work in teams, vol. 2, Greenwich, CT, JAI Press, 1995, p. 229-253. 
J. Kugelmass, Telecommuting: a Manager's Guide to Flexible Work Arrangements, 1995. W. H. Jr. Leach, "Virtual Company", A Unique Partnership in The Uinta Basin, April 2-6, 2002.

J. Lipnack, J. Stamps, Virtual teams: Reaching across space, time, and organizations with technology, New York, NY, John Wiley and Sons, Inc, 1997.

C. Loebbecke, T. Troelsen, “Towards Web-Based Investment Banking”, Electronic Journal of Organization Virtualness, ${ }^{\circ} 2(1), 2000$, p. 1-15, www.virtual-organization.net

H. C. Lucas, J. Baroudi, "The role of information technology in organization design", Journal of Management Information Systems, $\mathrm{n}^{\circ}$ 10(4), 1994, p. 9-23.

J. Magretta, "The power of virtual integration: An interview with Dell Computer's Michael Dell”, Harvard Business Review, n 76(2), Boston, 1998, p. 72-84.

S. A. Morris, T. Marshall, R. K. Rainer, "Impact of User Satisfaction and Trust on Virtual Team Members", Information resources Management Journal, April-June 2002, p. 22-30.

N. Nohria, R. G. Eccles, "Face-to-face: Making network organizations work", Networks and organizations, N. Nohria, R.G. Eccles (Eds.), Boston, MA, Harvard Business School Press, 1992, p. 288-308.

J. W. Palmer, C. Speier, "A Typology of Virtual Organizations: an empirical study", Proceedings of the Association for Information systems, J. Gupta (ed.), 1997 America conference, Indianapolis, 1997.

A. Perlo, C. Hills, « Réunir et souder une équipe virtuelle », L'Expansion Management Review, mars 1998, p. 114-119.

A. Penzias, "New Paths to Success", Fortune, n 131(11), 1995, p. 90-94.

A. M. Porter, "The Virtual Corporation: Where is it ?", Purchasing, March 23, 2000, p. 40-48.

M. E. Porter, Competitive Advantage: Creating and Sustaining Superior Performance, New York, The Free Press, 1985.

C. K. Prahalad, G. Hamel, "The Core Competence of the Corporation", Harvard Business Review, May-June 1990, p. 79-91.

J. F. Rayport, J. J. Sviokla, "Exploiting the Virtual Value Chain”, Harvard Business Review, November-December 1995, p. 75-85.

G. Sandhoff, "Virtual Organizations as Power-asymmetrical Networks", dans: P. Sieber, J. Griese (eds.), Organizational Virtualness and Electronic Commerce, Proceedings of the VoNet-Workshop, Simowa Verlag Bern, 1999, p. 103-119, www.virtual-organization.net W. Shen, "Virtual Organizations in Collaborative Design and Manufacturing Systems", Electronic Journal of Organization Virtualness, ${ }^{\circ} 2(2), 2000$, p. 43-57, www.virtual-organization.net

D. J. Skyrme, “The Realities of Virtuality”, Organizational Virtualness, P. Sieber, J. Griese (eds.), Proceedings of the VoNet-Workshop, Simowa Verlag Bern, 1998, p. 25-34, www.virtual-organization.net

C. C. Snow, S. A. Snell, S. C. Davison, "Use transnational teams to globalize your company”, Organizational Dynamics, n² 24(4), 1996, p. 50-67. 
G. Stalk, P. Evans, L. E. Shulman, "Competing on Capabilities: The New Rules of Corporate Strategy”, Harvard Business Review, March-April 1992, p. 57-69.

A. M. Townsend, S. M. DeMarie, A. R. Hendrickson, "Virtual teams: Technology and the workplace of the future", The Academy of Management Executive, $\mathrm{n}^{\circ}$ 12(3), 1998, p. 17-32. A. Wassenaar, "Understanding and Designing Virtual Organization Form", Virtual-organization.net, Newsletter $n^{\circ} 3(1), 1999$, p. 6-18, www.virtual-organization.net

B. M. Wiesenfeld, S. Raghuram, R. Garud, "Communication Patterns as Determinants of Organizational Identification in a Virtual Organization”, Journal of Computer-Mediated Communication, $\mathrm{n}^{\circ}$ 3(4), 1998. http://www.ascusc.org/jcmc/vol3/issue4/wiesenfeld.html U. Wiesenfeld, O. Fisscher, A. Pearson, K. Brockhoff, "Managing technology as a virtual enterprise", R\&D Management, $\mathrm{n}^{\circ}$ 31(3), 2001, p. 323-334.

R. Wigand, A. Picot, R. Reichwald, Information, Organization and Management: Expanding Markets and Corporate Boundaries, John Wiley \& Sons Ltd., Chichester, 1997. 